\title{
ON STRONGLY WRIGHT-CONVEX STOCHASTIC PROCESSES
}

\author{
DAWID KOTRYS
}

\begin{abstract}
Some characterizations of strongly Wright-convex stochastic processes are presented. Furthermore, the stochastic version of a theorem on strongly $\mathrm{J}$-convex functions majorized by strongly J-concave functions is given.
\end{abstract}

\section{Introduction}

In 1980 K. N i k o d e m [7] introduced the notion of convex (Jensen-convex) stochastic processes and proved some basic properties of them. In particular, he gave conditions under which Jensen-convex stochastic processes are continuous. Next, A. Sk ow roński in [9] and [10] obtained some further properties of Jensen-convex and Wright-convex stochastic processes.

Strongly convex stochastic processes were investigated by the author in [2]. The aim of this note is to introduce the notion of strongly Wright-convex stochastic processes and to present some properties of them. In particular, we give a characterization of strongly Wright-convex stochastic processes, which is a counterpart of the celebrated C. T. N g's representation theorem [5] of Wright-convex functions. We prove also the theorem on Jensen-convex stochastic processes majorized by Jensen-concave stochastic processes. It is a stochastic version of the results on (strongly) midconvex functions with (strongly) midconcave bounds proved in [1], 3], 6] and [8].

Let $(\Omega, \mathcal{A}, P)$ be an arbitrary probabilistic space. A function $X: \Omega \rightarrow \mathbb{R}$ is called a random variable, if it is $\mathcal{A}$-measurable. Given an interval $I$, a function $X: I \times \Omega \rightarrow \mathbb{R}$ is called a stochastic process, if for every $t \in I$ the function $X(t, \cdot)$ is a random variable.

Let $C: \Omega \rightarrow \mathbb{R}$ denote a positive random variable. We say that a stochastic process $X: I \times \Omega \rightarrow \mathbb{R}$ is

(C) 2016 Mathematical Institute, Slovak Academy of Sciences.

2010 Mathematics Subject Classification: Primary 26A51; Secondary 60G99.

Keywords: strongly convex stochastic processes, Wright-convex stochastic processes. 
(i) strongly convex with modulus $C(\cdot)$, if

$$
\begin{aligned}
& X(\lambda u+(1-\lambda) v, \cdot) \\
& \left.\quad \leqslant \lambda X(u, \cdot)+(1-\lambda) X(v, \cdot)-C(\cdot) \lambda(1-\lambda)(u-v)^{2} \quad \text { (a.e. }\right)
\end{aligned}
$$

for all $\lambda \in[0,1]$ and $u, v \in I$,

(ii) strongly midconvex (or strongly Jensen-convex) with modulus $C(\cdot)$, if the above inequality is assumed only for $\lambda=\frac{1}{2}$ and all $u, v \in I$, i.e.,

$$
X\left(\frac{u+v}{2}, \cdot\right) \leqslant \frac{1}{2} X(u, \cdot)+\frac{1}{2} X(v, \cdot)-\frac{C(\cdot)}{4}(u-v)^{2} \quad \text { (a.e.) }
$$

(iii) strongly Wright-convex with modulus $C(\cdot)$, if

$$
\begin{aligned}
& X(\lambda u+(1-\lambda) v, \cdot)+X((1-\lambda) u+\lambda v, \cdot) \\
& \quad \leqslant X(u, \cdot)+X(v, \cdot)-2 C(\cdot) \lambda(1-\lambda)(u-v)^{2} \quad \text { (a.e.) }
\end{aligned}
$$

holds for all $\lambda \in[0,1]$ and $u, v \in I$.

Obviously, by omitting the term $C(\cdot) \lambda(1-\lambda)(u-v)^{2}$ in cases (i) and (iii), we immediately get the definition of a convex, or Wright-convex stochastic processes introduced by K. Nikodem in [7, and A. Skowroński in [10], respectively. Simple computation shows that every strongly convex stochastic process is strongly Wright-convex, and every strongly Wright-convex stochastic process is strongly midconvex with the same modulus, but not the converse. More properties of strongly convex and strongly midconvex stochastic processes can be found in 2].

A stochastic process $A: \mathbb{R} \times \Omega \rightarrow \mathbb{R}$ is called additive if

$$
A(u+v, \cdot)=A(u, \cdot)+A(v, \cdot) \quad \text { (a.e.), }
$$

for all $u, v \in \mathbb{R}$. This definition was introduced by B. N a g y (see 44).

A stochastic process $X: I \times \Omega \rightarrow \mathbb{R}$ is called continuous in the interval $I$, if for all $t_{0} \in I$ we have

$$
P-\lim _{t \rightarrow t_{0}} X(t, \cdot)=X\left(t_{0}, \cdot\right),
$$

where $P$ - lim denotes the limit in probability. The notion was introduced by B. $\mathrm{Nagy}$ in [4].

\section{Strongly Wright-convex stochastic processes}

In [10] A. Skowroński proved that a stochastic process $X: I \times \Omega \rightarrow \mathbb{R}$ defined on an open interval $I$ is Wright-convex if and only if it can be presented in the form $X=X_{1}+A$, where $X_{1}$ is a convex stochastic process and $A$ is an additive process. It is a stochastic version of the celebrated theorem of C. T. N g [5] 


\section{ON STRONGLY WRIGHT-CONVEX STOCHASTIC PROCESSES}

characterizing Wright-convex functions. In this section we will give a counterpart of those results for strongly Wright-convex stochastic processes. We start with the following lemma.

Lemma 1. If a stochastic process $X: I \times \Omega \rightarrow \mathbb{R}$ defined on an open interval $I$ is convex and strongly midconvex with modulus $C(\cdot)$, then it is strongly convex with the same modulus.

Proof. Since $X$ is convex, then by K. Nikodem's result [7, Theorem 5] we arrive that $X$ is continuous. Using of [2, Theorem 7], we infer that $X$ is strongly convex with modulus $C(\cdot)$.

Theorem 2. Let $I$ be an open interval. A process $X: I \times \Omega \rightarrow \mathbb{R}$ is strongly Wright-convex with modulus $C(\cdot)$ if and only if there exist a stochastic process $X_{1}: I \times \Omega \rightarrow \mathbb{R}$ strongly convex with modulus $C(\cdot)$ and an additive stochastic process $A: \mathbb{R} \times \Omega \rightarrow \mathbb{R}$ such that

$$
X(u, \cdot)=X_{1}(u, \cdot)+A(u, \cdot) \quad \text { (a.e.), } \quad u \in I .
$$

Pr o of. For the proof of the "only if" part, we assume that $X$ is strongly Wright-convex with modulus $C(\cdot)$. Then $X$ is also Wright-convex and by A. Skowr oński theorem (see [10]) it can be represented in the following form

$$
X(u, \cdot)=X_{1}(u, \cdot)+A(u, \cdot) \quad \text { (a.e.), } u \in I,
$$

where $X_{1}: I \times \Omega \rightarrow \mathbb{R}$ is a convex stochastic process and $A: \mathbb{R} \times \Omega \rightarrow \mathbb{R}$ is an additive stochastic process. Since $X$ is strongly Wright-convex with modulus $C(\cdot)$, simple computation shows that the process $X-A$ is also strongly Wright-convex with modulus $C(\cdot)$, and consequently it is strongly midconvex with modulus $C(\cdot)$. By Lemma 1 the process $X_{1}(u, \cdot)=X(u, \cdot)-A(u, \cdot)$ (a.e.) is strongly convex with modulus $C(\cdot)$, which proves that $X$ has the representation (11). The "if" part is obvious.

By Lemma 2 proved in 2, Theorem 2 can be written in the following way.

Corollary 3. By Theorem 2 a strongly Wright-convex stochastic process $X: I \times \Omega \rightarrow \mathbb{R}$ with modulus $C(\cdot)$ has representation (1) with a strongly convex with modulus $C(\cdot)$ process $X_{1}: I \times \Omega \rightarrow \mathbb{R}$ and an additive process $A: \mathbb{R} \times \Omega \rightarrow \mathbb{R}$. By mentioned above Lemma 2 from [2] the process $X_{1}$ is of the form

$$
X(u, \cdot)=X_{2}(u, \cdot)+C(\cdot) u^{2}+A(u, \cdot) \quad \text { (a.e.), } \quad u \in I .
$$

P r o o f. By Theorem 2 the strongly Wright-convex stochastic process with modulus $C(\cdot)$ has the representation (11). By the mentioned above Lemma 2 (see [2]) every strongly convex stochastic process $X_{1}$ with modulus $C(\cdot)$ is of the form

$$
X_{1}(u, \cdot)=X_{2}(u, \cdot)+C(\cdot) u^{2} \quad \text { (a.e.) } \quad u \in I,
$$




\section{DAWID KOTRYS}

where $X_{2}: I \times \Omega \rightarrow \mathbb{R}$ is a convex stochastic process. Using (11) and (3) we get (2). The converse part is trivial.

Remark 4. An analogous characterization of strongly Wright-convex function with modulus $c$ was obtained by N. Merentes, K. Nikodem and S. Rivas in 3 .

\section{Strongly midconvex stochastic processes with strongly midconcave bounds}

It is known that if a midconvex function $f$ is bounded from above by a midconcave function $g$ then $f$ is Wright-convex and $g$ is Wright-concave. Moreover, there can be found a convex function $f_{1}$, a concave function $g_{1}$ and an additive function $a$ such that $f=f_{1}+a$ and $g=g_{1}+a$ (see [1], [6] and [6]). In 1995 A. Skowroński proved analogous theorem for J-convex and J-concave stochastic processes (see [9]). In this section we will present a counterpart of these results for strongly midconvex and strongly midconcave stochastic processes. We say that a stochastic process $X: I \times \Omega \rightarrow \mathbb{R}$ is strongly concave (strongly midconcave) with modulus $C(\cdot)$ if $-X$ is strongly convex (strongly midconvex) with modulus $C(\cdot)$.

Theorem 5. Let $I$ be an open interval. Assume that $X: I \times \Omega \rightarrow \mathbb{R}$ is strongly midconvex with modulus $C(\cdot), Y: I \times \Omega \rightarrow \mathbb{R}$ is strongly midconcave with modulus $C(\cdot)$ and $X(u, \cdot) \leqslant Y(u, \cdot)$ (a.e.) for every $u \in I$. Then there exist an additive stochastic process $A: \mathbb{R} \times \Omega \rightarrow \mathbb{R}$, a continuous stochastic process $X_{1}: I \times \Omega \rightarrow \mathbb{R}$ strongly convex with modulus $C(\cdot)$ and a continuous stochastic process $Y_{1}: I \times$ $\Omega \rightarrow \mathbb{R}$ strongly concave with modulus $C(\cdot)$ such that

and

$$
X(u, \cdot)=X_{1}(u, \cdot)+A(u, \cdot) \quad \text { (a.e.) }
$$

$$
Y(u, \cdot)=Y_{1}(u, \cdot)+A(u, \cdot) \quad \text { (a.e.) }
$$

for all $u \in I$.

P r o of. Since $X$ and $Y$ are strongly midconvex and strongly midconcave with modulus $C(\cdot)$, respectively, then $X$ and $Y$ are midconvex and midconcave, respectively. By A. Skow roński's theorem (see [9]) there exist the following representations for $X$ and $Y$

and

$$
X(u, \cdot)=X_{1}(u, \cdot)+A(u, \cdot) \quad \text { (a.e.) }
$$

$$
Y(u, \cdot)=Y_{1}(u, \cdot)+A(u, \cdot) \quad(\text { a.e. })
$$




\section{ON STRONGLY WRIGHT-CONVEX STOCHASTIC PROCESSES}

for $u \in I$, where $X_{1}: I \times \Omega \rightarrow \mathbb{R}$ is a convex stochastic process, $Y_{1}: I \times$ $\Omega \rightarrow \mathbb{R}$ is a concave stochastic process and $A: \mathbb{R} \times \Omega \rightarrow \mathbb{R}$ is an additive stochastic process. By the assumptions the additivity of the process $A$ we get that the process $X_{1}(u, \cdot)=X(u, \cdot)-A(u, \cdot)$ (a.e.) is strongly midconvex with modulus $C(\cdot)$ and the process $Y_{1}(u, \cdot)=Y(u, \cdot)-A(u, \cdot)$ (a.e.) is strongly midconcave with modulus $C(\cdot)$. Finally, by Lemma $1 X_{1}$ is strongly convex with modulus $C(\cdot)$ and $Y_{1}$ is strongly concave with modulus $C(\cdot)$. It proves that $X$ and $Y$ have the representation (4) with $X_{1}, Y_{1}$ and $A$ satisfies the desired properties. The continuity of $X_{1}$ and $Y_{1}$ follows from K. N i k o d e m's theorem (see [7]).

Remark 6. In the deterministic case the above theorem reduces to the result obtained in [3] for strongly midconvex functions with strongly midconcave bounds.

As before, by [2, Theorem 5 and Lemma 2], we get the following corollary.

Corollary 7. Let $I$ be an open interval. Assume that $X: I \times \Omega \rightarrow \mathbb{R}$ is strongly midconvex with modulus $C(\cdot), Y: I \times \Omega \rightarrow \mathbb{R}$ is strongly midconcave with modulus $C(\cdot)$ and $X(u, \cdot) \leqslant Y(u, \cdot)$ (a.e.) for every $u \in I$. Then there exist an additive stochastic process $A: \mathbb{R} \times \Omega \rightarrow \mathbb{R}$, a continuous, convex stochastic process $X_{2}: I \times \Omega \rightarrow \mathbb{R}$ and a continuous, concave stochastic process $Y_{2}: I \times \Omega \rightarrow \mathbb{R}$ such that

$$
\begin{array}{ll}
X(u, \cdot)=X_{2}(u, \cdot)+C(\cdot) u^{2}+A(u, \cdot) & \text { (a.e.), } \\
Y(u, \cdot)=Y_{2}(u, \cdot)+C(\cdot) u^{2}+A(u, \cdot) & \text { (a.e.), }
\end{array}
$$

for all $u \in I$.

The proof is similar to the proof of Corollary 3 , so we omit it.

\section{REFERENCES}

[1] KOMINEK, Z.: On a problem of K. Nikodem, Arch. Math. (Basel) 50 (1988), 287-288.

[2] KOTRYS, D.: Remarks on strongly convex stochastic processes, Aequationes Math. 86 (2012), 91-98.

[3] MERENTES, N.-NIKODEM, K.-RIVAS, S.: Remarks on strongly Wright-convex functions, Ann. Polon. Math. 102 (2011), 271-278.

[4] NAGY, B.: On a generalization of the Cauchy equation, Aequationes Math. 10 (1974), $165-171$.

[5] NG, C. T.: Functions generating Schur-convex sums, in: General Inequalities 5, Oberwolfach, 1986, Internat. Ser. Number. Math., Vol. 80, Birkhäuser, Basel, 1987, pp. 433-438.

[6] NG, C. T.: On midconvex functions with midconcave bounds, Proc. Amer. Math. Soc. 102 (1988), 538-540.

[7] NIKODEM, K.: On convex stochastic processes, Aequationes Math. 20 (1980), 184-197. 


\section{DAWID KOTRYS}

[8] NIKODEM, K.: Midpoint convex functions majorized by midpoint concave functions, Aequationes Math. 32 (1987), 45-51.

[9] SKOWROŃSKI, A.: On some properties of J-convex stochastic processes, Aequationes Math. 44 (1992), 249-258.

[10] SKOWROŃSKI, A.: On Wright-convex stochastic processes, Ann. Math. Sil. 9 (1995), $29-32$.

Received April 11, 2015

Department of Mathematics University of Bielsko-Biata

Willowa 2

PL-43-309 Bielsko-Biata

POLAND

E-mail: dkotrys@gmail.com 